\title{
RECOMBINANT HUMAN FGF18 PRESERVES DEPTH-DEPENDENT MECHANICAL INHOMOGENEITY IN ARTICULAR CARTILAGE
}

\author{
G.R. Meloni ${ }^{1,2,}$, A. Farran ${ }^{1, \S}$, B. Mohanraj ${ }^{1,2,3}$, H. Guehring ${ }^{4}$, R. Cocca ${ }^{1}$, E. Rabut ${ }^{1}$, R.L. Mauck ${ }^{1,2,3}$ \\ and G.R. Dodge $\mathrm{e}^{1,2,5}$ \\ ${ }^{1}$ McKay Orthopaedic Research Laboratory, Department of Orthopaedic Surgery, \\ Perelman School of Medicine, University of Pennsylvania, Philadelphia, PA 19104, USA \\ ${ }^{2}$ Translational Musculoskeletal Research Center, Corporal Michael J. Crescenz VA \\ Medical Centre, Philadelphia, PA 19104, USA \\ ${ }^{3}$ Department of Bioengineering, School of Engineering and Applied Science, \\ University of Pennsylvania, Philadelphia, PA 19104, USA \\ ${ }^{4}$ Merck KGaA, Darmstadt, Germany \\ ${ }^{5}$ Department of Otorhinolaryngology, Perelman School of Medicine, \\ University of Pennsylvania, Philadelphia, PA 19104, USA \\ $\S$ These authors contributed equally
}

\begin{abstract}
Articular cartilage is a specialised tissue that has a relatively homogenous endogenous cell population but a diverse extracellular matrix (ECM), with depth-dependent mechanical properties. Repair of this tissue remains an elusive clinical goal, with biological interventions preferred to arthroplasty in younger patients. Osteochondral transplantation (OCT) has emerged for the treatment of cartilage defects and osteoarthritis. Fresh allografts stored at $4{ }^{\circ} \mathrm{C}$ have been utilised, though matrix and cell viability loss remains an issue. To address this, several studies have developed media formulations to maintain cartilage explants in vitro. One promising factor for these applications is sprifermin, a human-recombinant fibroblast growth factor-18, which stimulates chondrocyte proliferation and matrix synthesis and is in clinical trials for the treatment of osteoarthritis. The study hypothesis was that addition of sprifermin during storage would maintain the unique depth-dependent mechanical profile of articular cartilage explants, a feature not often evaluated. Explants were maintained for up to 6 weeks with or without a weekly $24 \mathrm{~h}$ exposure to sprifermin (100 ng/ $\mathrm{mL}$ ) and the compressive modulus was assessed. Results showed that sprifermin-treated samples maintained their depth-dependent mechanical profile through 3 weeks, whereas untreated samples lost their mechanical integrity over 1 week of culture. Sprifermin also affected ECM balance by maintaining the levels of extracellular collagen and suppressing matrix metalloproteinase production. These findings support the use of sprifermin as a medium additive for OCT allografts during in vitro storage and present a potential mechanism where sprifermin may impact a functional characteristic of articular cartilage in repair strategies.
\end{abstract}

Keywords: Articular cartilage, fibroblast growth factor-18, mechanical properties.

*Address for correspondence: George R. Dodge, PhD, Department of Orthopaedic Surgery, Perelman School of Medicine, University of Pennsylvania, 110A Stemmler Hall, $36^{\text {th }}$ Street and Hamilton Walk, Philadelphia, PA 19104, USA.

Telephone number: +1 2155731514 Email: gdodge@pennmedicine.upenn.edu

Copyright policy: This article is distributed in accordance with Creative Commons Attribution Licence (http://creativecommons.org/licenses/by-sa/4.0/).

\section{Introduction}

The function of articular cartilage (AC) is to resist compressive loads generated during normal physical activity. In order to accomplish this task over a lifetime of use, the tissue has evolved to possess complex mechanical properties, including biphasic interactions between the solid and fluid phases of the tissue (Ateshian et al., 1994; Mow et al., 1980), tension-compression non-linearity - imbued by the high concentration and direction-dependent organisation of collagen (Huang et al., 2003; Mow et al., 1992; Park et al., 2003; Soltz and Ateshian, 2000; Sophia Fox et al., 2009) - and depth-dependent compressive properties - largely dictated by the high concentration and distribution of proteoglycans 
through its depth (Chen et al., 2001; Korver et al., 1990). In degenerative conditions, this complex distribution of the extracellular matrix (ECM) is disrupted and mechanical function is compromised, making the AC more isotropic and less specialised.

Despite its complex function over a lifetime of normal use, cartilage function can be compromised by acute and localised injuries. For instance, a recent review revealed that treatment of focal AC lesions has increased $5 \%$ annually, with an incidence rate of 90 per 10,000 patients (McCormick et al., 2014). When symptomatic, these focal injuries can be treated by chondroplasty, cell-based strategies - such as autologous chondrocyte implantation (ACI), matrixinduced autologous chondrocyte implantation (MACI) and microfracture - or osteochondral transfer (OCT), wherein an entire osteochondral unit is transferred from one location to another (Bartlett et al., 2005; Basad et al., 2010; Brittberg, 2008; Brittberg, 2010; Horas et al., 2003; Knutsen et al., 2007; Knutsen et al., 2004; Mithoefer et al., 2005; Peterson et al., 2010; Steadman et al., 2001). OCT can be performed in an autologous (where living donor tissue is moved from one location to another in an individual) or allogeneic fashion (where fresh cadaveric tissue is the source of the donor segment) (Aubin et al., 2001; Bugbee and Convery, 1999; Chow et al., 2004; Chu et al., 1999; Hangody et al., 2010; Hangody and Fules, 2003; Hangody et al., 2008; Marcacci et al., 2007; Williams et al., 2007). While early versions of this procedure used fresh-frozen and devitalised cadaveric tissue, recent studies have suggested that outcomes for allogeneic OCT are better when a living implant is used (Malinin et al., 2006; McCarty et al., 2010).

While promising as a therapeutic, donor osteochondral units must first be screened for communicable disease prior to implantation, necessitating storage of tissues for several weeks. Several studies have examined long-term storage of allografts at freezing $\left(-70^{\circ} \mathrm{C}\right)$, refrigerated $\left(4^{\circ} \mathrm{C}\right)$ and body $\left(37^{\circ} \mathrm{C}\right)$ temperatures, with the most common primary outcome being chondrocyte viability (Allen et al., 2005; Ball et al., 2004; Ohlendorf et al., 1996; Pallante-Kichura et al., 2013; Pallante et al., 2009; Pallante et al., 2012; Williams et al., 2004; Williams et al., 2003). Storage of allografts in hypothermic conditions may maintain the integrity of the ECM but does so at the expense of chondrocyte viability. While maintaining viability is readily achieved over such a period when storing grafts at body temperature, this storage and screening process is detrimental to the mechanical properties of the osteochondral unit, potentially limiting its efficacy upon implantation.

In vitro culture or storage of living AC result in rapid loss of mechanical function (Bian et al., 2008). This occurs because of the rapid loss and or degradation of the dense ECM (Hascall et al., 1983b). Once removed from the load-bearing synovial environment, both mechanical and biochemical cues that would normally promote tissue homeostasis are lost and the tissue begins to degrade. There have been numerous attempts to preserve or promote AC phenotype during in vitro culture of living AC, with varying degrees of success. Early studies in this field, employing mechanical loading systems and supplementation of media with growth factors, have demonstrated an anabolic effect and have explicated many of the key factors that regulate AC homeostasis (Fitzgerald et al., 2004; Guilak et al., 1994; Hall et al., 1991; Hascall et al., 1983a; Luyten et al., 1988; Sah et al., 1994; Sah et al., 1989). Towards the practical application of preserving implant properties prior to OCT procedures, these media formulations have been increasingly well defined, with some making their way to clinical and commercial application (Mickevicius et al., 2015; Teng et al., 2008). For instance, based on a chemically defined media formulation containing transforming growth factor-beta 3, the Missouri Osteochondral Preservation System enables the transfer of very large and highly viable osteochondral segments for total joint restoration (Garrity et al., 2012; Kuroki et al., 2017; Stoker et al., 2017; Stoker et al., 2018).

Despite this progress in the field, it is not yet clear whether such preservation systems fully retain the graded and refined mechanical properties of native tissue. It is also not clear whether the current media formulations represent the optimal formulation and whether other molecules may have a preservative effect. To that end, studies have recently shown that fibroblast growth factor 18 (FGF18) stimulates chondrocyte proliferation and matrix production in vitro and reduces AC degeneration and increases de novo matrix formation by osteoarthritic AC in vivo (Ellsworth et al., 2002; Moore et al., 2005). A recombinant version of this protein, known as sprifermin, currently a non-approved drug candidate and in clinical development for osteoarthritis treatment, also has positive effects in vitro, in vivo and in several recent pre-clinical and clinical trials in humans (Dahlberg et al., 2016; Gigout et al., 2017; Lohmander et al., 2014; Mori et al., 2014; Power et al., 2014; Reker et al., 2017). Sprifermin decreases collagen type I expression in monolayer culture and decreases collagen type II expression at dosing concentrations $>100 \mathrm{ng} / \mathrm{mL}$. With regards to cell morphology, treatment with sprifermin results in chondrocytes acquiring a more rounded morphology, with a loss of their elongated shape and stress fibres (Gigout et al., 2017). The present study tested whether the addition of sprifermin (recombinant FGF18) to standard media formulations could maintain the refined mechanical properties of AC during long-term in vitro culture, with a focus on the characteristic depth-dependent compressive properties of the native tissue.

\section{Materials and Methods}

\section{AC explant harvest and culture}

Full thickness AC explants were harvested from the trochlear groove of juvenile (3-6-month-old) bovine 
Table 1. Numbers for various assays.

\begin{tabular}{|c|c|c|c|c|c|c|c|c|}
\hline & & Collagen & GAG & $\begin{array}{c}\text { Solid } \\
\text { volume }\end{array}$ & MMP & $\begin{array}{c}\text { Media } \\
\text { GAG }\end{array}$ & $\begin{array}{c}\text { Normalised } \\
\text { bulk } \\
\text { modulus }\end{array}$ & $\begin{array}{c}\text { Local } \\
\text { mechanics }\end{array}$ \\
\hline Week 0 & Control & 23 & 23 & 19 & & & 23 & 15 \\
\hline \multirow{2}{*}{ Week 1} & Control & 26 & 26 & 17 & 25 & 24 & 21 & 16 \\
\hline & Sprifermin & 24 & 25 & 15 & 25 & 25 & 19 & 14 \\
\hline \multirow{2}{*}{ Week 2} & Control & 26 & 26 & 15 & 21 & 21 & 20 & 15 \\
\hline & Sprifermin & 27 & 27 & 17 & 21 & 21 & 21 & 16 \\
\hline \multirow{2}{*}{ Week 3} & Control & 30 & 30 & 20 & 17 & 17 & 25 & 16 \\
\hline & Sprifermin & 31 & 31 & 21 & 17 & 17 & 24 & 17 \\
\hline \multirow{2}{*}{ Week 4} & Control & 19 & 19 & 19 & 13 & 13 & 19 & 15 \\
\hline & Sprifermin & 19 & 19 & 19 & 13 & 13 & 17 & 13 \\
\hline \multirow{2}{*}{ Week 5} & Control & 19 & 19 & 19 & 10 & 10 & 18 & 14 \\
\hline & Sprifermin & 19 & 19 & 19 & 10 & 10 & 17 & 13 \\
\hline \multirow{2}{*}{ Week 6} & Control & 23 & 23 & 18 & 7 & 7 & 21 & 12 \\
\hline & Sprifermin & 24 & 24 & 19 & 7 & 7 & 24 & 15 \\
\hline
\end{tabular}

stifle joints (Research 87, Boylston, MA, USA) in aseptic conditions and using a sterile $4 \mathrm{~mm}$ diameter biopsy punch. Explants were washed three times in sterile phosphate buffered saline (PBS) supplemented with 200 units $/ \mathrm{mL}$ penicillin, $200 \mu \mathrm{g} / \mathrm{mL}$ streptomycin and $0.5 \mu \mathrm{g} / \mathrm{mL}$ amphotericin B (Gibco). Following washing, explants were sharply dissected to remove non-cartilaginous tissues (subchondral bone) and cultured overnight in complete medium consisting of Dulbecco's modified Eagle medium (DMEM; Gibco), $10 \%$ foetal bovine serum, 100 units/mL of penicillin, $100 \mu \mathrm{g} / \mathrm{mL}$ of streptomycin, $2.5 \mu \mathrm{g} / \mathrm{mL}$ of amphotericin B (Gibco), 1× MEM Vitamins (Corning), $25 \mathrm{mM}$ 4-(2-hydroxyethyl)-1-piperazineethanesulphonic acid (HEPES) buffer (Gibco) and $50 \mu \mathrm{g} / \mathrm{mL}$ L-ascorbic acid 2-phosphate (Sigma-Aldrich). Following overnight culture, samples were randomly assigned to treatment and time groups. Samples were maintained fully submerged in $1 \mathrm{~mL}$ complete medium and cultured at $37^{\circ} \mathrm{C}$ and $5 \% \mathrm{CO}_{2}$. Medium was changed three times per week, with $1 \mathrm{~mL}$ collected at each medium change frozen at $-80^{\circ} \mathrm{C}$ for later analysis. The initial study was planned for a duration of 3 weeks with follow up studies planned for end points between 3 and 6 weeks. Data presented were gleaned from all samples at the same time points over multiple experiments. Replicate numbers are provided with each figure in addition to the summarised data in Table 1. Reduction in sample number for matrix metalloproteinase (MMP) and media glycosaminoglycan (GAG) assessment was the result of sequential tissue removal at each weekly time point.

Recombinant human FGF18 (rhFGF18) treatment $100 \mathrm{ng} / \mathrm{mL}$ rhFGF18 (alias sprifermin, Merck $\mathrm{KGaA}$ ) was added to complete medium. Sprifermin concentration was based on previous in vitro assays demonstrating a dose dependency in a wide range of concentrations (Gigout et al., 2017). A treatment duration of $24 \mathrm{~h}$ each week was chosen to enhance the 'hit and run' effect that sprifermin has been shown to have, whereas short-term exposure elicits the greatest anabolic response. After treatment, medium was replaced with fresh complete medium (Gigout et al., 2017). Controls were treated similarly, with exchange of complete medium as well after $24 \mathrm{~h}$. Thereafter, medium in all cultures was changed every $3 \mathrm{~d}$.

\section{Mechanical testing}

For unconfined compression testing, samples were tested using a custom-built device (Fig. 1) (Mauck et al., 2000; Mauck et al., 2006). Briefly, a $0.02 \mathrm{~N}$ creep load was applied at $10 \mu \mathrm{m} / \mathrm{s}$ and held for $300 \mathrm{~s}$, followed by $10 \%$ compressive strain applied over $200 \mathrm{~s}$ followed by a 1,000 s hold. Equilibrium modulus was calculated by dividing the measured load at the end of the 1,000 s hold by the cross-sectional area and applied strain. To assay the depth-dependent mechanical properties of the AC explants, samples were tested in unconfined compression using a custom $\mu \mathrm{m}$-driven inverted microscope mounted apparatus (Farrell et al., 2012). The deep zone of each sample was trimmed on a freezing-stage microtome to ensure parallel surfaces for mechanical testing, while still preserving the superficial zone. Then, samples were measured with digital callipers and cut diametrically to produce a semi-cylinder. While this may cause some fibre discontinuity in the sample, all specimens were tested in the same fashion. One half was fixed in $4 \%$ paraformaldehyde for histology and the other half was stained with Hoechst 33342 (1: 100 dilution) to identify cell nuclei that would later be used as fiducial markers. Then, the tissue 
was placed cut-side-down into the device and the loading platens were brought into contact with the sample. Fluorescent images were acquired on an inverted microscope (Nikon Eclipse TE2000-U) and equilibrium load readings were recorded at initial contact and following each of five $4 \%$ strain increments, up to $20 \%$ strain. Samples were loaded manually using a $\mu \mathrm{m}$-driven linear stage at approximately $1 \%$ strain/s and held for 800 s. Then, an image correlation software (Vic2d, Correlated Solutions) was used to compute a 2D strain field with a resolution of $16.25 \mu \mathrm{m}$ throughout the depth of the tissue using the nuclei as fiducial markers (Farrell et al., 2012). Data were further analysed using a custom MATLAB program to calculate the average strain and the direction of loading in $50 \mu \mathrm{m}$ bins across the depth of the sample, starting at the AC surface and continuing through the depth of the sample. Strain data from the first and last $100 \mu \mathrm{m}$ of each sample were discarded due to edge effects. The equilibrium modulus for each bin was calculated by dividing the equilibrium load by the measured cross-sectional area and bin strain. This resulted in a depth-dependent profile of the compressive equilibrium modulus. To compare these local data to the tissue-scale modulus, acquired using the test described above, the average strain across the whole tissue was calculated. Then, these data were normalised by dividing the measured modulus at each time point by the average modulus at the start of each experiment. This was done to account for differences in the testing modalities and biological variability between studies. Next, these data were included in the analysis of the tissue-scale mechanics.
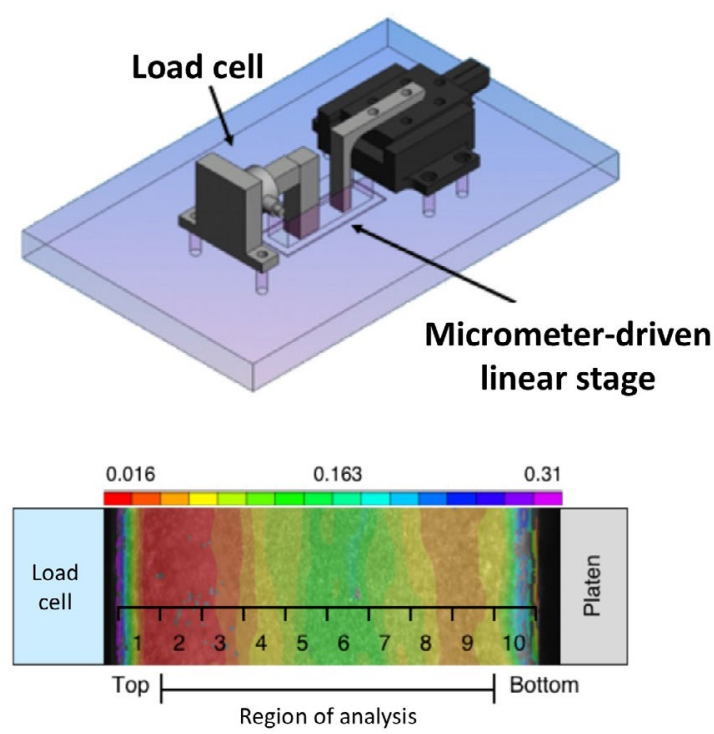

Fig. 1. Schematic of custom compression device mounted on an inverted fluorescent microscope and heat map of strain mapped to ten distinct regions of analysis from the superficial to deep zone. Adapted from Farrell et al. (2012), used with permission.

\section{Biochemical assays}

Sulphated GAG content was quantified using the colorimetric 1,9-dimethylmethylene blue assay (Farndale et al., 1986). Collagen content was quantified using the orthohydroxyproline assay and a conversion factor of 7.6 (Stegemann and Stalder, 1967). MMP activity levels in the media were quantified with the SensoLyte Fluroimetric assay (AnaSpec) using a fluorometric assay reading at exposure $490 \mathrm{~nm}$, emission $520 \mathrm{~nm}$. The assay kit is designed to detect MMP-1, 2, 3, 7, 8, 9, 12, 13 and 14 , making it ideal for high throughput screening and detection of generic MMP activity. Wet and dry weights were recorded before samples were digested for $18 \mathrm{~h}$ at $60{ }^{\circ} \mathrm{C}$ in proteinase $\mathrm{K}$ digestion buffer (50 units/mL in $100 \mathrm{mM}$ Tris- $\mathrm{HCl}$; Worthington, Lakewood, NJ, USA), with frequent mixing. GAG and collagen content were quantified on digested samples. GAG and MMP levels in the medium were quantified on a weekly basis. Solid volume fraction was calculated as the quotient of the dry weight divided by the wet weight of each sample. From this, the change in water content was expressed as a percentage by calculating the difference in solid volume fraction at each week relative to the mean week 0 value.

\section{Data analysis and statistics}

For mechanical testing data analysis, studies were normalised to mean time zero values and combined. GAG and collagen content were normalised to wet weight to account for variability in the size of each individual tissue segment. Error bars in figures represent the standard error of the mean.

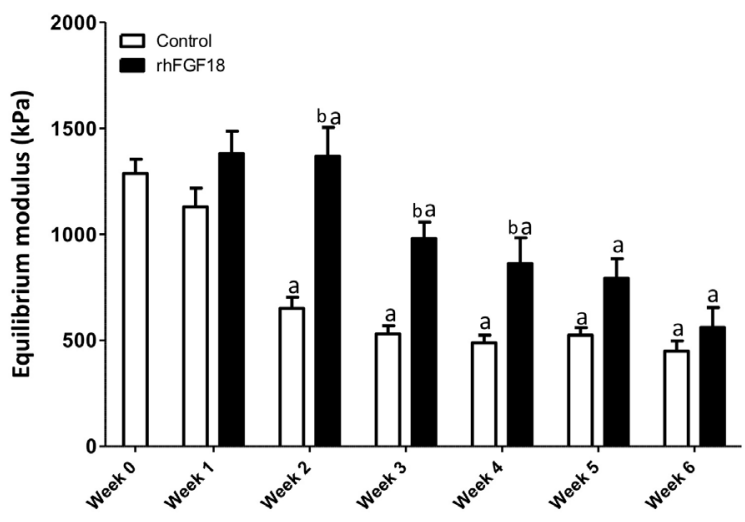

Fig. 2. Equilibrium modulus of AC on the tissue scale. Samples were cultured in vitro for up to 6 weeks $\pm 100 \mathrm{ng} / \mathrm{mL}$ rhFGF18. Treated explants did not differ from baseline modulus throughout 5 weeks. Additionally, the modulus of the treated explants was significantly higher than that of the control at weeks 2,3 and 4 ( ${ }^{\mathrm{a}} p<0.05$ vs. week 0 , b $p<0.05$ vs. control; $n=17-25)$. 
Statistical significance was determined using 1-way or 2-way ANOVA with Bonferroni's post-hoc test, as appropriate.

\section{Results}

AC explant tissue-scale mechanics with FGF18 treatment

The equilibrium modulus of AC explants decreased during in vitro culture over 6 weeks, regardless of sprifermin treatment (Fig. 2). Samples cultured in control medium (Fig. 2, white bars) had a significantly lower equilibrium modulus as compared to week 0 values at every week following 1 week of culture $(n=12-23, p<0.05)$. Conversely, the equilibrium modulus of samples treated with rhFGF18 (black bars) did not become significantly lower until week $6(n=17-29, p<0.05)$. Similarly, the tissue-scale equilibrium modulus of the explants treated with rhFGF18 was higher than control explants at 2, 3 and 4 weeks $(n=12-29, p<0.05)$.

\section{AC explant local mechanics with sprifermin treatment}

To better understand these differences in tissue-scale compressive mechanics, each sample was tested using a custom device to assay tissue modulus in $50 \mu \mathrm{m}$ increments from the superficial zone through the deep zone. Baseline, or week 0, depth-dependent mechanics showed an increase in modulus as function of distance from the AC surface (Fig. 3, grey), consistent with previous findings (Schinagl et al., 1996; Wang et al., 2003; Wang et al., 2002). Control samples (dashed lines, $n=12-17$ ) had moduli that were below baseline levels throughout the depth of the AC explants. These differences became more pronounced with culture duration. Additionally, control samples quickly lost the depth-dependence characteristics of native $\mathrm{AC}$, seen as a flattening of the modulus profile. In contrast, sprifermin-treated samples (solid coloured lines, $n=12-17$ ) retained a depth-dependent profile, matching that of the baseline week 0 control through 5 weeks of in vitro culture, with modulus increasing as a function of distance from the AC surface. Further investigations were performed in the $100-1,000 \mu \mathrm{m}$ region of the tissue, in order to determine the differences in the superficial and lower/middle zone of the AC samples, rather than the full depth (Fig. 3). This layer, spanning $1 \mathrm{~mm}$ in thickness, represents the AC layer that will remain in the adult. Trends were quantified as average moduli in regions near the surface (100$150 \mu \mathrm{m})$, in the middle $(450-500 \mu \mathrm{m})$ and in the deep $(950-1,000 \mu \mathrm{m})$ zones of the tissue as a function of culture duration (Fig. 4). At these distances from the AC surface, control samples (dashed lines) showed a decreased modulus by week 3 near the surface and in the middle zone and by week 4 in the deep zone. rhFGF18-treated samples (solid lines) showed no differences in the deep zone, a decrease at week 4 in the middle zone and decreases at weeks 4 and 5
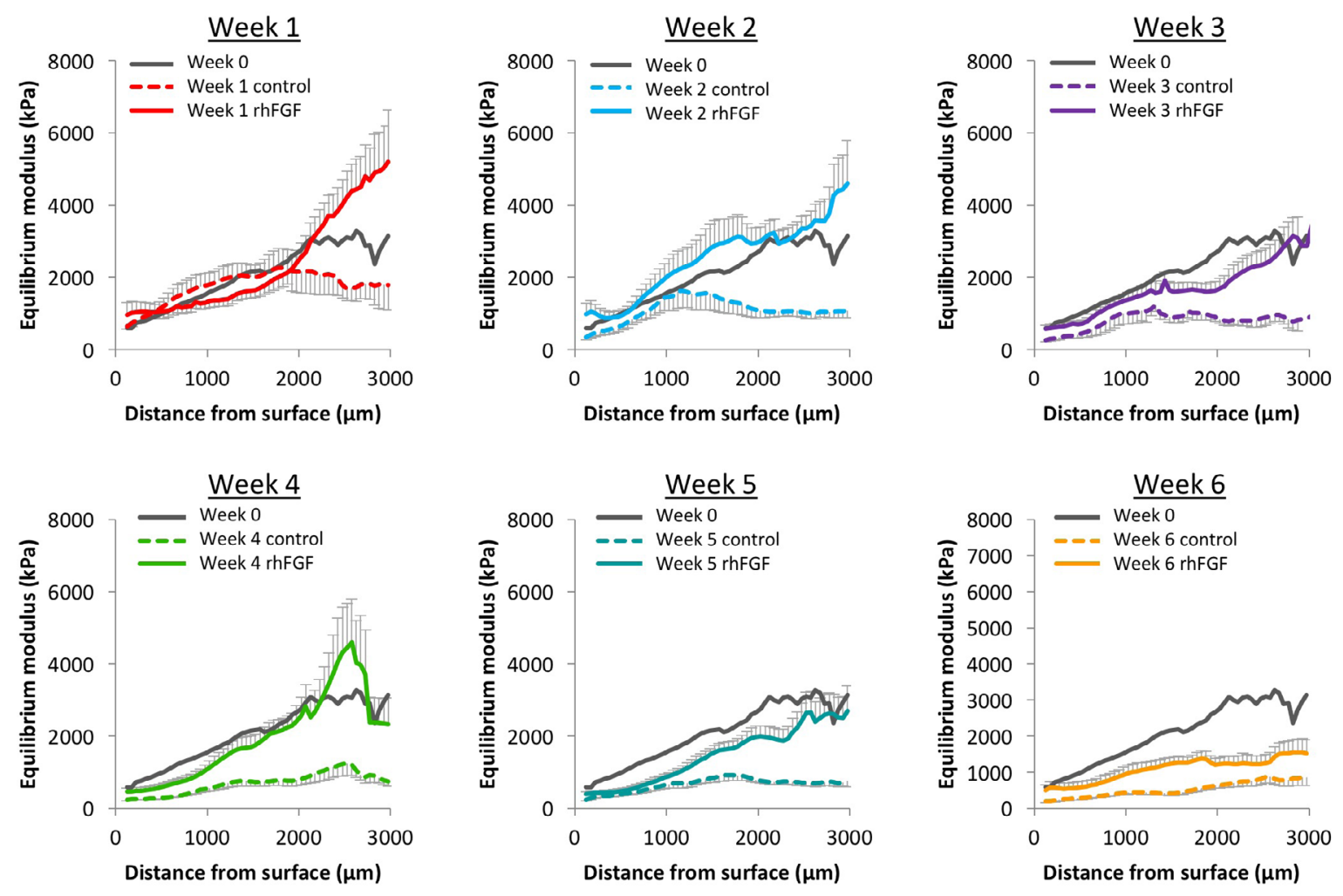

Fig. 3. Depth-dependent equilibrium modulus of AC explants cultured in vitro for 0-6 weeks. Baseline modulus through the depth is shown in grey $(n=12-17)$. 
near the surface. Overall, control samples showed a decrease in modulus in 11 out of 18 samples (61\%), whereas sprifermin samples presented differences in only 3 out of 18 samples (17\%).

Biochemical content of sprifermin-treated explant Following mechanical testing, the biochemical composition of the tested samples was determined. Biochemical composition was determined on the half that was mechanically tested. The adjacent hemi-cylinder was fixed in $4 \%$ paraformaldehyde. Total collagen content, as a percentage of wet weight, decreased significantly in control samples after 1 week of culture (Fig. 5a, white bars) as compared to week 0 levels $(n=19-30, p<0.05)$. In comparison, sprifermin-treated explants (solid bars) had significantly lower collagen content after only 4 and 6 weeks of in vitro culture $(n=19-30, p<0.05)$. Additionally, sprifermin-treated explants had higher collagen content as compared to controls at all time points after week 2. GAG content was also measured as a percentage of wet weight in time (Fig. 5b). GAG content significantly decreased for control samples starting at week 2 (white bars), while for sprifermintreated explants these decreases were not apparent until week 3. Only at week 2, was a difference in GAG content detectable between groups.

\section{Explant macroscopic appearance and water content with sprifermin treatment}

Through 6 weeks, explants in control conditions appeared to visibly swell and distort from their original cylindrical shape, whereas sprifermintreated explants maintained their size and shape. Both control and sprifermin treatment groups showed a significant difference in solid volume fraction after 1 week of culture (Fig. 6a). However, the solid volume fraction with sprifermin treatment was higher than controls at each following week. This can be expressed as a change in water content (Fig. 6b), where water content significantly increased in control samples at all time points. For treated explants, while there was an increase after week 1 , this change was attenuated as compared to that observed in control conditions.

\section{Matrix loss in explants treated with sprifermin} Assays on the medium were also performed to determine GAG and MMP release from the tissue. Although there were some minor differences in
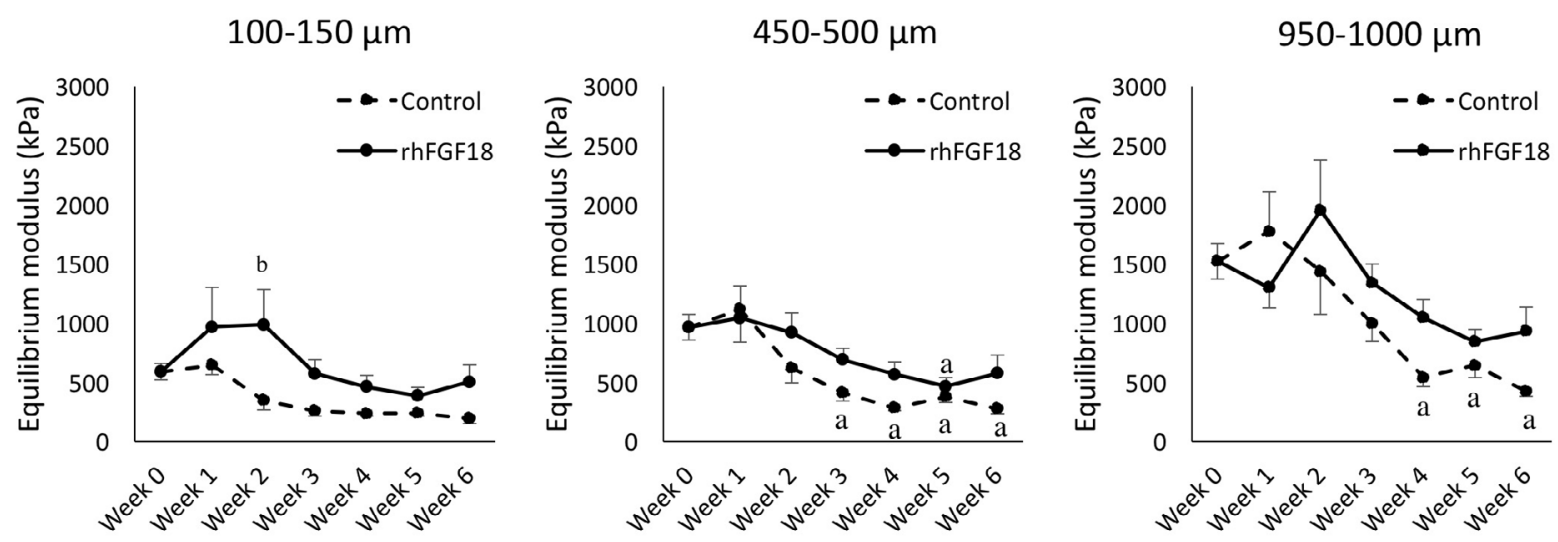

Fig. 4. Mean equilibrium modulus of AC explants at distances of 100-150, 450-500 and 950-1,000 $\mu \mathrm{m}$ from the AC surface over the course of 6 weeks of in vitro culture. ${ }^{\mathrm{a}} p<0.05$ vs. week $0,{ }^{\mathrm{b}} p<0.05$ vs. control.
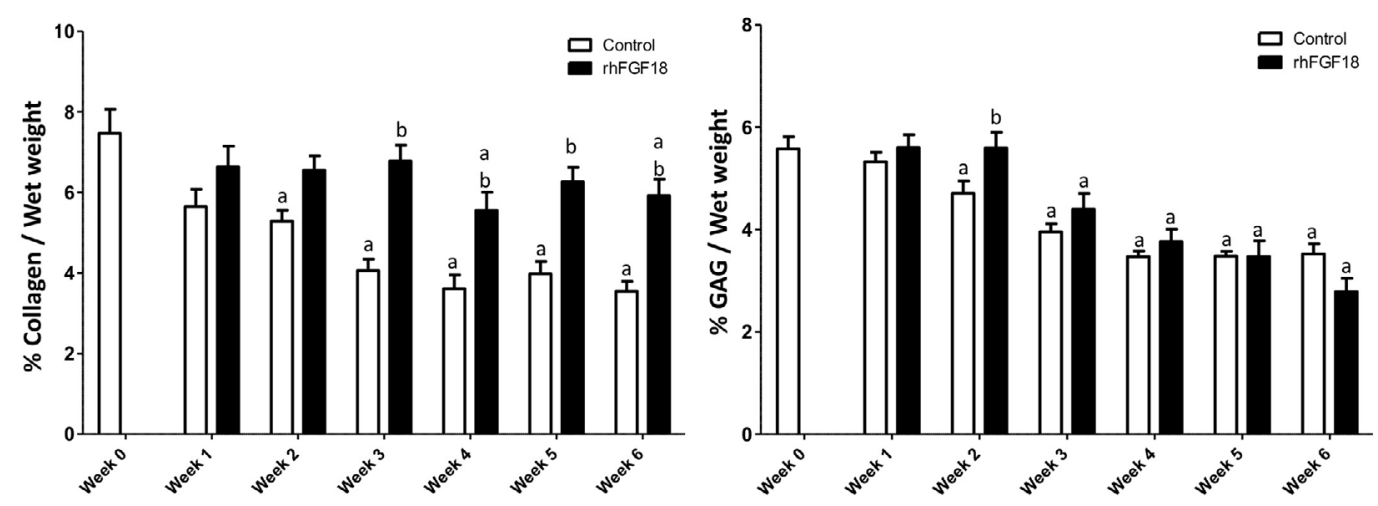

Fig. 5. Collagen content as measured by the orthohydroxyproline assay and sulphated-GAG contented measured by the 1,9-dimethylmethylene blue assay for AC explants cultured in vitro for 0-6 weeks. Data were normalised to wet weight. ${ }^{a} p<0.05$ vs. week 0 control, ${ }^{b} p<0.05$ vs. control at same time point $(n=19-31)$. 
weekly GAG released into the medium, none of these differences was statistically significant (Fig. 7a). Similarly, cumulative GAG release into the medium did not differ between treatment groups (Fig. 7b). Control samples also showed a steady level of MMP released into the medium each week. Conversely, sprifermin-treated samples showed a marked reduction in MMP in the medium through the first 3 weeks of culture (Fig. 8).

\section{Discussion}

The efficacy of sprifermin as storage media additive to preserve AC during extended in vitro storage was demonstrated. At the tissue level, sprifermin had a significant effect on the equilibrium modulus, which quickly decreased in control samples starting after week 2, while no changes were observed in sprifermin-treated samples through 5 weeks of in vitro culture. Additionally, sprifermin-treated group had a higher equilibrium modulus when compared to control samples at weeks 2-4. These findings were replicated using two different measurement techniques; while not directly comparable, the differences between treated and untreated samples were reproducibly similar. To visualise the order of magnitude of these changes, the results from one study are shown in Fig. 2. In this replicate study, sprifermin maintained or increased the tissue-scale modulus from week 0 through week 2 of culture, whereas control explants decreased in modulus at all time points.

To further examine and identify changes, a technique to track strain (and calculate modulus) throughout the depth of the AC tissue was utilised, allowing the determination of the depth-dependent mechanical properties of each sample. This is an important feature, given that the native surrounding tissue showed such depth dependence; consequently, functional integration of the implanted osteochondral unit might be compromised if the implant does not match native tissue values. This high-resolution method resulted in a plot of modulus of $50 \mu \mathrm{m}$
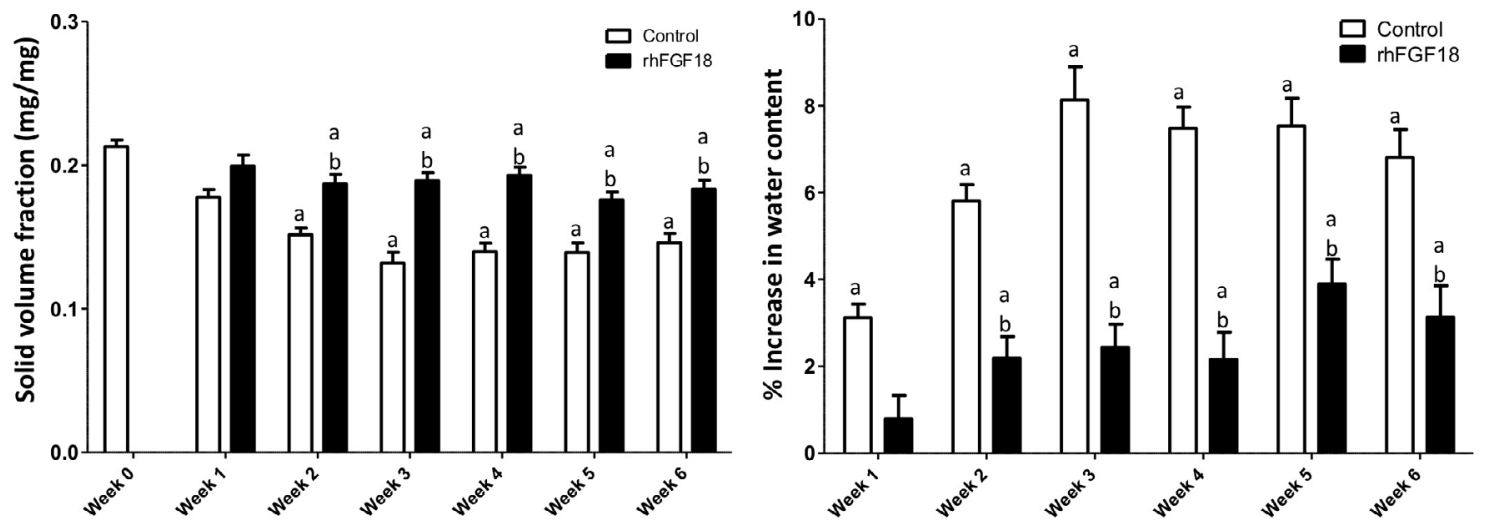

Fig. 6. Increase in water content is attenuated with sprifermin treatment. ${ }^{a} p<0.05$ vs. week 0 control, ${ }^{\mathrm{b}} p<0.05$ vs. control at same time point $(n=19-31)$.
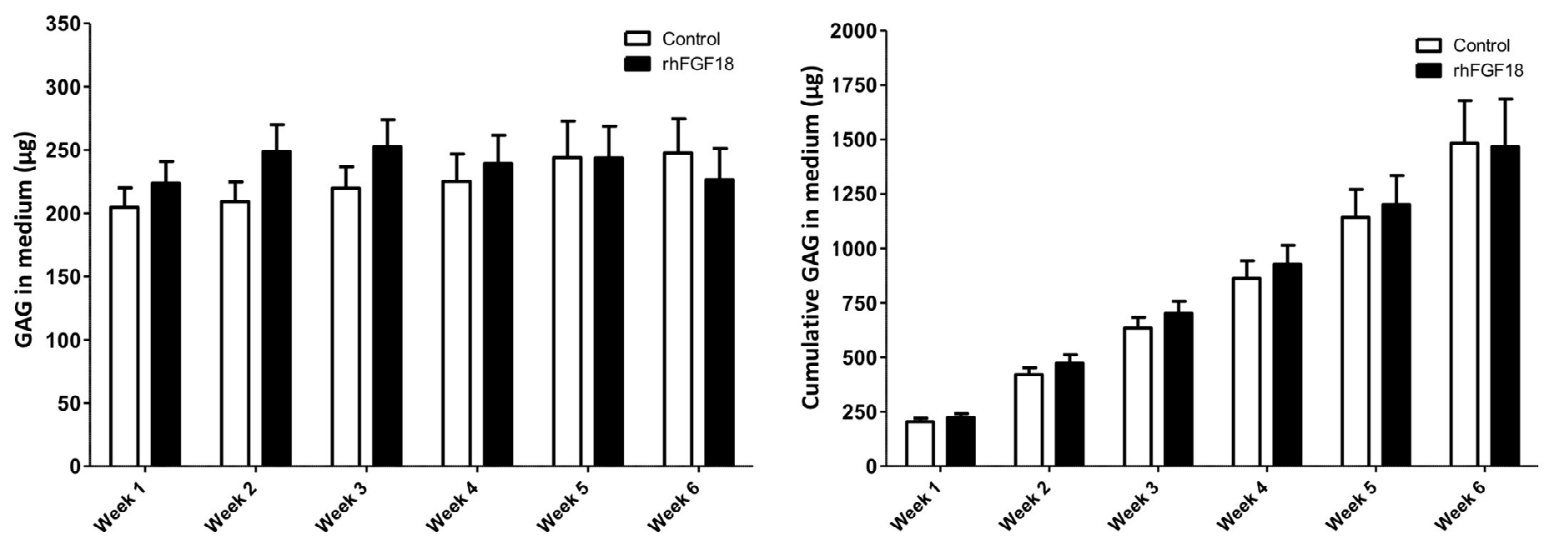

Fig. 7. GAG released into the culture medium per explant and cumulative GAG in the medium over the course of 6 weeks of in vitro culture $(n=7-25)$. 


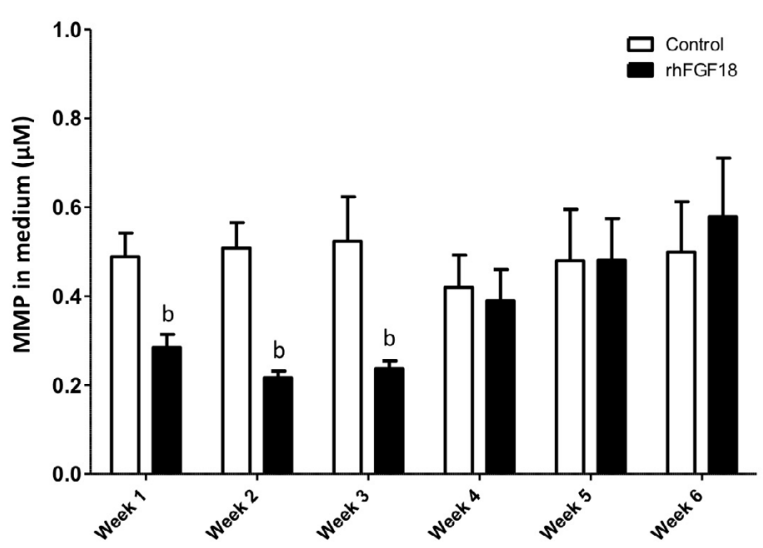

Fig. 8. MMP concentration in the medium. MMP release into the medium was significantly suppressed with weekly sprifermin treatments through the first 3 weeks of exposure $\left({ }^{b} p<0.05 v s\right.$. control at same time point, $n=7-25)$.

increments. As early as 1 week of in vitro culture, a divergence in mechanical properties was observed between control (Fig. 3, dashed lines) and sprifermin samples (Fig. 3, solid lines). Such mechanical properties decreased in control samples at 2,000 to $3,000 \mu \mathrm{m}$ from the AC surface and increased in sprifermin samples at the same depth. This divergence point moved closer to the AC surface as the duration of culture increased, indicating that control samples were losing their mechanical properties in the deep zones faster than in the superficial zones. This pattern resulted in control samples that lost their intrinsic inhomogeneity, whereas samples from the sprifermin treatment group maintained a depth-dependent modulus profile through 5 weeks of culture.

The largest differences in local mechanical properties were observed in the deepest zones of the samples, furthest from the AC surface. Samples used were from immature bovine $A C$, whereas when the animal is fully mature the $\mathrm{AC}$ will be much thinner. Thus, it was important to examine changes in this tissue closer to the AC surface, in regions that will likely remain as mature, permanent $\mathrm{AC}$ and not develop into bone. In these more superficial regions, sprifermin also preserved the mechanical properties of the AC for at least 3 weeks (Fig. 4). This finding demonstrated that sprifermin could preserve the depth-dependent mechanical characteristics of AC in an ex vivo environment for an extended duration. Given that this 3-week time window was sufficient for screening the implants for communicable diseases, this finding may improve preservation methods for allogeneic OCT procedures.

The mechanical testing, carried out to determine both tissue-scale and local properties of the tissue, consisted of uniaxial compression. This is a sensible first assay, given that native tissue functions in compression and testing of the tissue in this way is necessary to understand the change in mechanical properties (Korhonen et al., 2003). There are two principal ECM components that regulate the mechanical properties of $\mathrm{AC}$, proteoglycans (PGs) and collagen (Julkunen et al., 2007). Although PG content resists compressive loads through chargecharge repulsion (through the fixed negative charges on the PG), water retention and interstitial fluid pressurisation (Khoshgoftar et al., 2013) (through the Donnan osmotic effect and frictional drag of fluid flowing through small pores), it appears that PGs are not the central player in the action of sprifermin. Rather, the preservation of $\mathrm{AC}$ mechanical integrity with weekly sprifermin treatment appears to be due to maintenance of the collagen network. While collagen acts to resist tensile loads, it is also a key contributor to the mechanical response in compression by resisting the outward expansion of the AC tissue (Poisson effect). The equilibrium Poisson ratio of native AC is quite low (in the order of 0.1-0.2), due in large part to the high tensile properties in the plane of the tissue because of the high collagen content (Wang et al., 2003). Explants treated with rhFGF18 had higher collagen levels when compared to control samples at all time points from week 3 to 6 . Additionally, treated samples did not differ from baseline levels until week 4. These significant differences were not seen in either GAG levels in the tissue itself or GAG measured in the medium. Given that the mechanical response of the tissue is largely governed by these two components, it was concluded that sprifermin maintained native AC mechanical properties through preservation of the collagen network.

Further evidence for the importance of collagen and the ability of sprifermin to maintain this crucial network was the solid volume fraction or tissue swelling during in vitro culture. Solid volume fraction and collagen content are directly related (Bank et al., 2000). The change in solid volume fraction can also be expressed as the influx of water. Control samples were unable to resist the imbibition of water into the tissue; consequently, the tissue swelled substantially. Since control samples were unable to resist this expansion, they might also lose their ability to undergo interstitial fluid pressurisation during loading, resulting in reduced mechanical functionality. AC samples from the sprifermin treatment group were better able to resist swelling as the collagen network was better preserved. Changes in collagen content were likely due to differential expression and activity of MMPs. MMP activity in the culture medium was measured as an indicator of MMP expression and activation during in vitro culture. Sprifermin treatment significantly suppressed MMP levels for 3 weeks, while control samples had a higher, steady concentration of MMPs in the media. During the same time frame, sprifermin treatment maintained tissue-scale mechanical properties, local depth-dependent mechanical properties and collagen content. The mechanism of action of FGF18 consists in the induction of cell proliferation. In the articular joint context, this translates into the specific proliferation of chondrocytes because of the FGF 
receptor subtype distribution. Chondrocytes need space for proliferating and, therefore, this process is started with the expression of matrix-dissolving proteases, e.g. MMP13. Chondrocytes that recently proliferated produce more matrix (more, because the $\mathrm{AC}$ is populated by more chondrocytes and more, because of an increased matrix production rate per cell) by activation of the ERK pathway (Gigout et al., 2017). In 3D culture, FGF18 increases the number of matrix-producing chondrocytes, improves type II : I collagen ratio and enables chondrocytes to produce a hyaline ECM. Furthermore, FGF18 displays a 'hit and run' mode of action, which is a common phenomenon for growth factors (Gigout et al., 2017). Chondrocyte survival time in AC explants seems to be increased in in vitro experiments; however, cell viability was not evaluated in the present study.

The findings of the study suggested that sprifermin may be used as a medium supplement during storage or extended culture of viable AC tissue, having a significant clinical application. Currently, donor tissue for allograft procedures is refrigerated while it is screened prior to transplant. This practice maintains some of the mechanical properties of the native tissue, but also results in low chondrocyte viability. Using sprifermin under normal culture and storage conditions, AC mechanical inhomogeneity can be maintained over a similar time course. AC from multiple bovine sources of approximately the same age was used in the study. The advantage was to reduce the number of variables, namely AC age. However, in future work and for clinical applications, AC maturity will be an important factor to consider as it has a great effect on the tissue homeostasis and activity. Studies to address this issue and to replicate these findings in human-sourced materials are currently underway.

\section{Conclusions}

Sprifermin preserved the intrinsic depth-dependent mechanical properties of AC during long-term in vitro culture/storage conditions through maintenance of extracellular collagen and suppression of MMP production. This methodology may be applied in a clinical scenario to improve on current transplantation practice, where, prior to surgery, the tissue is stored during its screening, compromising its mechanical properties. Furthermore, the study presented potential mechanisms for sprifermin mode of action in protecting the integrity of AC and, ultimately, impacting a functional characteristic of AC in restorative strategies.

\section{Acknowledgements}

The study was in part supported by a grant from Merck KGaA of which one of the authors (H.G.) is a paid employee. Support was also provided by the
Department of Veteran's Affairs (I01 RX001213) and the NIH through the Penn Centre for Musculoskeletal Diseases Histology and Biomechanics Cores (P30 AR069619).

\section{References}

Allen RT, Robertson CM, Pennock AT, Bugbee WD, Harwood FL, Wong VW, Chen AC, Sah RL, Amiel D (2005) Analysis of stored osteochondral allografts at the time of surgical implantation. Am J Sports Med 33: 1479-1484.

Ateshian GA, Lai WM, Zhu WB, Mow VC (1994) An asymptotic solution for the contact of two biphasic cartilage layers. J Biomech 27: 1347-1360.

Aubin PP, Cheah HK, Davis AM, Gross AE (2001) Long-term followup of fresh femoral osteochondral allografts for posttraumatic knee defects. Clin Orthop Relat Res 391 Suppl: S318-327.

Ball ST, Amiel D, Williams SK, Tontz W, Chen AC, Sah RL, Bugbee WD (2004) The effects of storage on fresh human osteochondral allografts. Clin Orthop Relat Res 418: 246-252.

Bank RA, Soudry M, Maroudas A, Mizrahi J, TeKoppele JM (2000) The increased swelling and instantaneous deformation of osteoarthritic cartilage is highly correlated with collagen degradation. Arthritis Rheum 43: 2202-2210.

Bartlett W, Skinner JA, Gooding CR, Carrington RW, Flanagan AM, Briggs TW, Bentley G (2005) Autologous chondrocyte implantation versus matrixinduced autologous chondrocyte implantation for osteochondral defects of the knee: a prospective, randomised study. J Bone Joint Surg Br 87: 640-645.

Basad E, Ishaque B, Bachmann G, Sturz H, Steinmeyer J (2010) Matrix-induced autologous chondrocyte implantation versus microfracture in the treatment of cartilage defects of the knee: a 2-year randomised study. Knee Surg Sports Traumatol Arthrosc 18: 519-527.

Bian L, Lima EG, Angione SL, Ng KW, Williams DY, Xu D, Stoker AM, Cook JL, Ateshian GA, Hung CT (2008) Mechanical and biochemical characterization of cartilage explants in serum-free culture. J Biomech 41: 1153-1159.

Brittberg M (2008) Autologous chondrocyte implantation - technique and long-term follow-up. Injury 39 Suppl 1: S40-49.

Brittberg M (2010) Cell carriers as the next generation of cell therapy for cartilage repair: a review of the matrix-induced autologous chondrocyte implantation procedure. Am J Sports Med 38: 12591271.

Bugbee WD, Convery FR (1999) Osteochondral allograft transplantation. Clin Sports Med 18: 67-75.

Chen AC, Bae WC, Schinagl RM, Sah RL (2001) Depth- and strain-dependent mechanical and electromechanical properties of full-thickness bovine articular cartilage in confined compression. J Biomech 34: 1-12. 
Chow JC, Hantes ME, Houle JB, Zalavras CG (2004) Arthroscopic autogenous osteochondral transplantation for treating knee cartilage defects: a 2- to 5-year follow-up study. Arthroscopy 20: 681-690.

Chu CR, Convery FR, Akeson WH, Meyers M, Amiel D (1999) Articular cartilage transplantation. Clinical results in the knee. Clin Orthop Relat Res 36: $159-168$.

Dahlberg LE, Aydemir A, Muurahainen N, Gühring H, Fredberg Edebo H, Krarup-Jensen N, Ladel CH, Jurvelin JS (2016) A first-in-human, double-blind, randomised, placebo-controlled, dose ascending study of intra-articular rhFGF18 (sprifermin) in patients with advanced knee osteoarthritis. Clin Exp Rheumatol 34: 445-450.

Ellsworth JL, Berry J, Bukowski T, Claus J, Feldhaus A, Holderman S, Holdren MS, Lum KD, Moore EE, Raymond F, Ren HP, Shea P, Sprecher C, Storey H, Thompson DL, Waggie K, Yao L,

Fernandes RJ, Eyre DR, Hughes SD (2002) Fibroblast growth factor-18 is a trophic factor for mature chondrocytes and their progenitors. Osteoarthritis Cartilage 10: 308-320.

Farndale RW, Buttle DJ, Barrett AJ (1986) Improved quantitation and discrimination of sulphated glycosaminoglycans by use of dimethylmethylene blue. Biochim Biophys Acta 883: 173-177.

Farrell MJ, Comeau ES, Mauck RL (2012) Mesenchymal stem cells produce functional cartilage matrix in three-dimensional culture in regions of optimal nutrient supply. Eur Cell Mater 23: 425-440.

Fitzgerald JB, Jin M, Dean D, Wood DJ, Zheng MH, Grodzinsky AJ (2004) Mechanical compression of cartilage explants induces multiple time-dependent gene expression patterns and involves intracellular calcium and cyclic AMP. J Biol Chem 279: $19502-$ 19511.

Garrity JT, Stoker AM, Sims HJ, Cook JL (2012) Improved osteochondral allograft preservation using serum-free media at body temperature. Am J Sports Med 40: 2542-2548.

Gigout A, Guehring H, Froemel D, Meurer A, Ladel C, Reker D, Bay-Jensen AC, Karsdal MA, Lindemann S (2017) Sprifermin (rhFGF18) enables proliferation of chondrocytes producing a hyaline cartilage matrix. Osteoarthritis Cartilage 25: 18581867.

Guilak F, Meyer BC, Ratcliffe A, Mow VC (1994) The effects of matrix compression on proteoglycan metabolism in articular cartilage explants. Osteoarthritis Cartilage 2: 91-101.

Hall AC, Urban JP, Gehl KA (1991) The effects of hydrostatic pressure on matrix synthesis in articular cartilage. J Orthop Res 9: 1-10.

Hangody L, Dobos J, Balo E, Panics G, Hangody LR, Berkes I (2010) Clinical experiences with autologous osteochondral mosaicplasty in an athletic population: a 17-year prospective multicenter study. Am J Sports Med 38: 1125-1133.

Hangody L, Fules P (2003) Autologous osteochondral mosaicplasty for the treatment of full-thickness defects of weight-bearing joints: ten years of experimental and clinical experience. J Bone Joint Surg Am 85-A Suppl 2: 25-32.

Hangody L, Vásárhelyi G, Hangody LR, Sükösd Z, Tibay G, Bartha L, Bodó G (2008) Autologous osteochondral grafting - technique and long-term results. Injury 39 Suppl 1: S32-39.

Hascall VC, Handley CJ, McQuillan DJ, Hascall GK, Robinson HC, Lowther DA (1983a) The effect of serum on biosynthesis of proteoglycans by bovine articular cartilage in culture. Arch Biochem Biophys 224: 206-223.

Hascall VC, Morales TI, Hascall GK, Handley CJ, McQuillan DJ (1983b) Biosynthesis and turnover of proteoglycans in organ culture of bovine articular cartilage. J Rheumatol Suppl 11: 45-52.

Horas U, Pelinkovic D, Herr G, Aigner T, Schnettler R (2003) Autologous chondrocyte implantation and osteochondral cylinder transplantation in cartilage repair of the knee joint. A prospective, comparative trial. J Bone Joint Surg Am 85-A: 185-192.

Huang CY, Soltz MA, Kopacz M, Mow VC, Ateshian GA (2003) Experimental verification of the roles of intrinsic matrix viscoelasticity and tensioncompression nonlinearity in the biphasic response of cartilage. J Biomech Eng 125: 84-93.

Julkunen P, Kiviranta P, Wilson W, Jurvelin JS, Korhonen RK (2007) Characterization of articular cartilage by combining microscopic analysis with a fibril-reinforced finite-element model. J Biomech 40: 1862-1870.

Khoshgoftar M, Wilson W, Ito K, van Donkelaar CC (2013) Influence of tissue- and cell-scale extracellular matrix distribution on the mechanical properties of tissue-engineered cartilage. Biomech Model Mechanobiol 12: 901-913.

Knutsen G, Drogset JO, Engebretsen L, Grøntvedt T, Isaksen V, Ludvigsen TC, Roberts S, Solheim E, Strand T, Johansen O (2007) A randomized trial comparing autologous chondrocyte implantation with microfracture. Findings at five years. J Bone Joint Surg Am 89: 2105-2112.

Knutsen G, Engebretsen L, Ludvigsen TC, Drogset JO, Grøntvedt T, Solheim E, Strand T, Roberts S, Isaksen V, Johansen O (2004) Autologous chondrocyte implantation compared with microfracture in the knee. A randomized trial. J Bone Joint Surg Am 86-A: 455-464.

Korhonen RK, Laasanen MS, Toyras J, Lappalainen R, Helminen HJ, Jurvelin JS (2003) Fibril reinforced poroelastic model predicts specifically mechanical behavior of normal, proteoglycan depleted and collagen degraded articular cartilage. J Biomech 36: 1373-1379.

Korver GH, van de Stadt RJ, van Kampen GP, van der Korst JK (1990) Composition of proteoglycans synthesized in different layers of cultured anatomically intact articular cartilage. Matrix 10: 394-401.

Kuroki K, Stoker AM, Stannard JP, Bozynski CC, Cook CR, Pfeiffer FM, Cook JL (2017) Biologic joint 
repair strategies: the Mizzou BioJoint story. Toxicol Pathol 45: 931-938.

Lohmander LS, Hellot S, Dreher D, Krantz EF, Kruger DS, Guermazi A, Eckstein F (2014) Intraarticular sprifermin (recombinant human fibroblast growth factor 18) in knee osteoarthritis: a randomized, double-blind, placebo-controlled trial. Arthritis Rheumatol 66: 1820-1831.

Luyten FP, Hascall VC, Nissley SP, Morales TI, Reddi AH (1988) Insulin-like growth factors maintain steady-state metabolism of proteoglycans in bovine articular cartilage explants. Arch Biochem Biophys 267: 416-425.

Malinin T, Temple HT, Buck BE (2006) Transplantation of osteochondral allografts after cold storage. J Bone Joint Surg Am 88: 762-770.

Marcacci M, Kon E, Delcogliano M, Filardo G, Busacca M, Zaffagnini S (2007) Arthroscopic autologous osteochondral grafting for cartilage defects of the knee: prospective study results at a minimum 7-year follow-up. Am J Sports Med 35: 2014-2021.

Mauck RL, Soltz MA, Wang CC, Wong DD, Chao PH, Valhmu WB, Hung CT, Ateshian GA (2000) Functional tissue engineering of articular cartilage through dynamic loading of chondrocyte-seeded agarose gels. J Biomech Eng 122: 252-260.

Mauck RL, Yuan X, Tuan RS (2006) Chondrogenic differentiation and functional maturation of bovine mesenchymal stem cells in long-term agarose culture. Osteoarthritis Cartilage 14: 179-189.

McCarty WJ, Pallante AL, Rone RJ, Bugbee WD, Sah RL (2010) The proteoglycan metabolism of articular cartilage in joint-scale culture. Tissue Eng Part A 16: 1717-1727.

McCormick F, Harris JD, Abrams GD, Frank R, Gupta A, Hussey K, Wilsen H, Bach B Jr, Cole B (2014) Trends in the surgical treatment of articular cartilage lesions in the United States: an analysis of a large private-payer database over a period of 8 years. Arthroscopy 30: 222-226.

Mickevicius T, Pockevicius A, Kucinskas A, Gudas R, Maciulaitis J, Noreikaite A, Usas A (2015) Impact of storage conditions on electromechanical, histological and histochemical properties of osteochondral allografts. BMC Musculoskelet Disord 16: 314. DOI: 10.1186/s12891-015-0776-y.

Mithoefer K, Williams RJ, Warren RF, Potter HG, Spock CR, Jones EC, Wickiewicz TL, Marx RG (2005) The microfracture technique for the treatment of articular cartilage lesions in the knee. A prospective cohort study. J Bone Joint Surg Am 87: 1911-1920.

Moore EE, Bendele AM, Thompson DL, Littau A, Waggie KS, Reardon B, Ellsworth JL (2005) Fibroblast growth factor-18 stimulates chondrogenesis and cartilage repair in a rat model of injury-induced osteoarthritis. Osteoarthritis Cartilage 13: 623-631.

Mori Y, Saito T, Chang SH, Kobayashi H, Ladel CH, Geuhring H, Chung UI, Kawaguchi H (2014) Identification of fibroblast growth factor-18 as a molecule to protect adult articular cartilage by gene expression profiling. J Biol Chem 289: 10192-10200.

Mow VC, Kuei SC, Lai WM, Armstrong CG (1980) Biphasic creep and stress relaxation of articular cartilage in compression? Theory and experiments. J Biomech Eng 102: 73-84.

Mow VC, Ratcliffe A, Poole AR (1992) Cartilage and diarthrodial joints as paradigms for hierarchical materials and structures. Biomaterials 13: 67-97.

Ohlendorf C, Tomford WW, Mankin HJ (1996) Chondrocyte survival in cryopreserved osteochondral articular cartilage. J Orthop Res 14: 413-416.

Pallante-Kichura AL, Chen AC, Temple-Wong MM, Bugbee WD, Sah RL (2013) In vivo efficacy of fresh versus frozen osteochondral allografts in the goat at 6 months is associated with PRG4 secretion. J Orthop Res 31: 880-886.

Pallante AL, Bae WC, Chen AC, Gortz S, Bugbee WD, Sah RL (2009) Chondrocyte viability is higher after prolonged storage at 37 degrees $C$ than at 4 degrees $C$ for osteochondral grafts. Am J Sports Med 37 Suppl 1: 24S-32S.

Pallante AL, Gortz S, Chen AC, Healey RM, Chase DC, Ball ST, Amiel D, Sah RL, Bugbee WD (2012) Treatment of articular cartilage defects in the goat with frozen versus fresh osteochondral allografts: effects on cartilage stiffness, zonal composition, and structure at six months. J Bone Joint Surg Am 94: 1984-1995.

Park S, Krishnan R, Nicoll SB, Ateshian GA (2003) Cartilage interstitial fluid load support in unconfined compression. J Biomech 36: 1785-1796.

Peterson L, Vasiliadis HS, Brittberg M, Lindahl A (2010) Autologous chondrocyte implantation: a longterm follow-up. Am J Sports Med 38: 1117-1124.

Power J, Hernandez P, Guehring H, Getgood A, Henson F (2014) Intra-articular injection of rhFGF-18 improves the healing in microfracture treated chondral defects in an ovine model. J Orthop Res 32: 669-676.

Reker D, Kjelgaard-Petersen CF, Siebuhr AS, Michaelis M, Gigout A, Karsdal MA, Ladel C, BayJensen AC (2017) Sprifermin (rhFGF18) modulates extracellular matrix turnover in cartilage explants ex vivo. J Transl Med 15: 250. DOI: 10.1186/s12967-0171356-8.

Sah RL, Chen AC, Grodzinsky AJ, Trippel SB (1994) Differential effects of bFGF and IGF-I on matrix metabolism in calf and adult bovine cartilage explants. Arch Biochem Biophys 308: 137-147.

Sah RL, Kim YJ, Doong JY, Grodzinsky AJ, Plaas AH, Sandy JD (1989) Biosynthetic response of cartilage explants to dynamic compression. J Orthop Res 7: 619-636.

Schinagl RM, Ting MK, Price JH, Sah RL (1996) Video microscopy to quantitate the inhomogeneous equilibrium strain within articular cartilage during confined compression. Ann Biomed Eng 24: 500-512.

Soltz MA, Ateshian GA (2000) A conewise linear elasticity mixture model for the analysis of tension- 
compression nonlinearity in articular cartilage. J Biomech Eng 122: 576-586.

Sophia Fox AJ, Bedi A, Rodeo SA (2009) The basic science of articular cartilage: structure, composition, and function. Sports Health 1: 461-468.

Steadman JR, Rodkey WG, Rodrigo JJ (2001) Microfracture: surgical technique and rehabilitation to treat chondral defects. Clin Orthop Relat Res 391 Suppl: S362-369.

Stegemann H, Stalder K (1967) Determination of hydroxyproline. Clin Chim Acta 18: 267-273.

Stoker AM, Stannard JP, Cook JL (2017) Chondrocyte viability at time of transplantation for osteochondral allografts preserved by the Missouri Osteochondral Preservation System versus standard tissue bank protocol. J Knee Surg 31: 772-780.

Stoker AM, Stannard JP, Kuroki K, Bozynski CC, Pfeiffer FM, Cook JL (2018) Validation of the Missouri Osteochondral Allograft Preservation System for the maintenance of osteochondral allograft quality during prolonged storage. Am J Sports Med 46: 58-65.

Teng MS, Yuen AS, Kim HT (2008) Enhancing osteochondral allograft viability: effects of storage media composition. Clin Orthop Relat Res 466: $1804-$ 1809.

Wang CC, Chahine NO, Hung CT, Ateshian GA (2003) Optical determination of anisotropic material properties of bovine articular cartilage in compression. J Biomech 36: 339-353.

Wang CC, Deng JM, Ateshian GA, Hung CT (2002) An automated approach for direct measurement of two-dimensional strain distributions within articular cartilage under unconfined compression. J Biomech Eng 124: 557-567.

Williams RJ, DreeseJC, Chen CT(2004)Chondrocyte survival and material properties of hypothermically stored cartilage: an evaluation of tissue used for osteochondral allograft transplantation. Am J Sports Med 32: 132-139.

Williams RJ, Ranawat AS, Potter HG, Carter T, Warren RF (2007) Fresh stored allografts for the treatment of osteochondral defects of the knee. J Bone Joint Surg Am 89: 718-726.

Williams SK, Amiel D, Ball ST, Allen RT, Wong VW, Chen AC, Sah RL, Bugbee WD (2003) Prolonged storage effects on the articular cartilage of fresh human osteochondral allografts. J Bone Joint Surg Am 85-A: 2111-2120.

\section{Discussion with Reviewers}

Neil Duncan: Did you measure cell viability of controls and treated samples, specifically as a function of depth?

Authors: No, this is an interesting point but it was not the subject of the present study. Due to the limited amount of tissue from the region to be studied, it was not possible to obtain enough samples for every test.

Russell Craddock: Can the authors comment on the ability of FGF18 to preserve AC micro-structure? Authors: This was not assayed in the present or other studies with the exception of the study by Farrell et al. (2012), where no marked changes are observed at an electron microscopic level of analysis.

Editor's note: The Scientific Editor responsible for this paper was Stephen Ferguson. 